\title{
INCIDÊNCIA DE Listeria monocytogenes EM QUEIJO DE COALHO REFRIGERADO PRODUZIDO INDUSTRIALMENTE
}

\author{
MARIA AURINEIDE DE ABREU CASTELO BRANCO* \\ EVÂNIA ALTINA TEIXEIRA DE FIGUEIREDO** \\ MARIA DE FÁTTIMA BORGES *** \\ MARIA CRISTINA DELGADO DA SILVA *** \\ MARIA TEREZA DESTRO *****
}

\begin{abstract}
Avaliou-se a incidência de Listeria monocytogenes em queijo de coalho, produzido industrialmente e comercializado sob refrigeração na cidade de FortalezaCE (Brasil). Também foram avaliadas as condições de $\mathrm{pH}$ e de atividade de água nas amostras contaminadas com espécies de Listeria. Foram analisadas 84 amostras de queijo de coalho industrializado de diferentes marcas empregando o TECRA Listeria Visual Immunoassay (LISVIA) modificado. Das 84 amostras, 16 (19\%) estavam contaminadas com Listeria monocytogenes, 5 $(5,9 \%)$ com Listeria innocua e $1(1,2 \%)$ com Listeria grayi. Listeria monocytogenes foi isolada na faixa de $\mathrm{pH}$ de 5,75 a 6,37 e em atividade de água entre 0,949 e 0,970 . O TECRA LISVIA detectou a presença de Listeria spp. em $9(10,7 \%)$ amostras. Todas as amostras positivas no teste rápido foram confirmadas por testes culturais e bioquímicos e em todas foi detectada a presença de Listeria monocytogenes. O plaqueamento das amostras negativas na leitura visual do teste rápido permitiu o isolamento de Listeria spp. em 8 amostras e em 7 foi detectada a presença de Listeria monocytogenes.
\end{abstract}

PALAVRAS-CHAVE: TECRA LISVIA; QUEIJO DE COALHO; Listeria Monocytogenes.

* Mestranda em Tecnologia de Alimentos, Universidade Federal do Ceará (UFC), Fortaleza, CE (e-mail: aurycb@hotmail.com).

** Professora, Doutora, Departamento de Tecnologia de Alimentos, UFC.

*** Pesquisadora da Embrapa Agroindústria Tropical, Fortaleza, CE.

**** Professora, Doutora, Departamento de Nutrição da Universidade Federal de Alagoas, Maceió, AL.

***** Professora, Doutora, Departamento de Alimentos e Nutrição Experimental da Universidade de São Paulo (USP), São Paulo, SP. 


\section{INTRODUÇÃO}

Dentre os queijos produzidos no Nordeste brasileiro, o queijo de coalho é o de maior tradição e um dos mais difundidos, apresentando especial consumo nos Estados do Ceará, Paraíba, Pernambuco e Rio Grande do Norte (BENEVIDES, 1999; AQUINO, 1983).

O queijo de coalho alcança grande popularidade no estado do Ceará, sendo consumido diariamente por $26,4 \%$ da população de Fortaleza e por $27,7 \%$ dos moradores do interior (SEBRAE, 1998).

Apesar da grande importância socioeconômica do queijo de coalho para a região Nordeste, muitas vezes o produto é elaborado sem que sejam observados os padrões higiênico-sanitários. Portanto, seu consumo pode oferecer consideráveis riscos à saúde pública. Diversas pesquisas realizadas no Nordeste denunciam as más condições higiênico-sanitárias do queijo de coalho (FEITOSA, 1984; SANTOS et al., 1995; FLORENTINO e MARTINS, 1999; MENDES et al., 1999; NASSU et al., 2000; BORGES et al., 2000; LEITE JR. et al., 2000).

Muitos alimentos crus e processados apresentam contaminação por L. monocytogenes, a qual pode ocorrer em qualquer etapa da cadeia alimentar (ROCOURT e COSSART, 1997). Sua natureza ubiqüitária, psicrófila e grande resistência a adversidades diferem a Listeria monocytogenes de muitos patógenos veiculados por alimentos.

Listeria monocytogenes já foi isolada de produtos lácteos, carnes, frangos e mariscos (BANNISTER, 1987; BECKERS et al.1987; DILLON e PATTEL, 1992; JOHNSON et al. 1990), bem como de grande variedade de produtos tipo "delicatessen" (RYSER e MARTH, 1991).

Os laticínios são particularmente suscetíveis à contaminação por Listeria monocytogenes e segundo WHER (1987) a contaminação desse grupo de alimentos deve-se, possivelmente, mais à contaminação cruzada que propriamente à excreção da bactéria no leite. Dentre os laticínios, os queijos são os mais estudados com relação à contaminação com L. monocytogenes, tendo sido incriminados em diversos casos de listeriose. GUERRA e BERNARDO (1999b) afirmam que a bactéria pode alcançar níveis de $10^{4}$ a $10^{7} \mathrm{UFC} / \mathrm{g}$ de queijo, cujos valores dependem do tipo de queijo e da tecnologia de fabricação empregada. 
Diversas pesquisas têm demonstrado a habilidade de Listeria monocytogenes em sobreviver e crescer durante a produção, maturação e estocagem de diferentes tipos de queijos (RYSER, MARTH e DOYLE, 1985; RYSER e MARTH, 1987a; RYSER e MARTH, 1987b).

A ingestão de Listeria monocytogenes pode desencadear uma séria doença humana, denominada listeriose (ROCOURT e COSSART, 1997; FARBER e PETERKIN, 1991; RYSER e MARTH, 1991), sendo o trato gastrintestinal sua porta de entrada no organismo. A listeriose, doença de origem alimentar atípica, desperta grande interesse em saúde pública. A síndrome caracteriza-se por um quadro de alta severidade e natureza não-entérica (meningite, septicemia, aborto). Entretanto, existem registros de surtos de listeriose caracterizados por sintomas gastrintestinais envolvendo indivíduos saudáveis (RIEDO et al., 1994; DALTON et al., 1997; GUERRA e BERNARDO, 1999b).

A taxa de mortalidade (incluindo aborto) pode atingir índices de $30 \%$, especialmente para o grupo de risco composto por mulheres grávidas (incluindo fetos), recém-nascidos, idosos e indivíduos com o sistema imunológico comprometido (ROCOURT e COSSART, 1997; PEREIRA e ROCOURT, 1993).

A maioria dos alimentos responsáveis por casos esporádicos e surtos de listeriose é produzida industrialmente, tratada termicamente em nível seguro e consumido sem prévio aquecimento (MCLAUCHLIN, 1996, apud FIGUEIREDO, 2000).

O presente estudo teve como objetivos avaliar a incidência de Listeria monocytogenes em queijo de coalho produzido industrialmente, correlacionar os valores de $\mathrm{pH}$ e Aw, bem como avaliar a eficiência do TECRA Listeria Visual Immunoassay na detecção de espécies de Listeria em queijo de coalho.

\section{MATERIAL E MÉTODOS}

\subsection{MATERIAL}

Foram analisadas 84 amostras de queijo de coalho de diferentes marcas, produzidas industrialmente e comercializadas sob refrigeração. As amostras foram coletadas ao acaso, em supermercados da cidade 
de Fortaleza, durante os meses de julho a dezembro de 2001. As 4 marcas de queijo de coalho avaliadas apresentavam o selo de inspeção governamental e foram transportadas até o laboratório, sob refrigeração, para análises microbiológicas, físicas e físico-químicas.

\subsection{ANÁLISE MICROBIOLÓGICA}

A avaliação presuntiva de Listeria spp. foi efetuada mediante teste rápido TECRA Listeria Visual Immunoassay, modificado pela substituição do caldo LEB (Listeria enrichment Broth) pelo caldo LEBUVM1 (OXOID). Para o enriquecimento secundário, 1,0 mL do LEBUVM 1 (após incubação) foi semeado em tubo contendo 9,0 mL de caldo Fraser (FB-OXOID).

\subsubsection{Detecção de Listeria spp.}

Após o enriquecimento das amostras, essas e os reagentes foram preparados conforme o protocolo do TECRA LISTERIA VISUAL IMMUNOASSAY (TECRA, 2000) aprovado pela Association of Official Anallytical Chemistry (AOAC) e pelo Ministério da Agricultura do Brasil.

\subsubsection{Isolamento e identificação de Listeria spp.}

O Isolamento e identificação de Listeria spp. foi realizado de acordo com metodologia recomendada pelo Health Protection Branch do Canadá (FARBER et al., 1994), após as etapas de enriquecimento para as 84 amostras analisadas.

\subsection{ANÁLISES FÍSICAS E FÍSICO-QUÍMICAS}

\subsection{1 $\mathrm{pH}$}

$\mathrm{O} \mathrm{pH}$ das amostras positivas para Listeria spp. foi determinado em medidor de $\mathrm{pH}$ (HANNA INSTRUMENTS, modelo HI 9321), calibrado com solução tampão de $\mathrm{pH}$ 4,0 e 7,0, segundo metodologia recomendada pela AOAC (1992).

\subsubsection{Atividade de água}

A determinação da atividade de água (Aa) das amostras positivas para 
Listeria spp. foi efetuada em aparelho digital Aqualab da Decagon Devices Inc. EUA, modelo CX-2 (AQUALAB, 2001).

\section{RESULTADOS E DISCUSSÃO}

3.1 INCIDÊNCIA DE Listeria spp. EM QUEIJO DE COALHO, VALORES DE pH E ATIVIDADE DE ÁGUA (Aw) DAS AMOSTRAS CONTAMINADAS

Das 84 amostras de queijo de coalho analisadas, 16 (19\%) estavam contaminadas com Listeria monocytogenes, 5 (5,9\%) com Listeria innocua e 1 (1,2\%) com Listeria grayi. Foram positivos para Listeria monocytogenes os queijos das marcas A, C e D. Das 25 amostras de queijo de coalho da marca $A$, apenas $1(4 \%)$ foi positiva para Listeria monocytogenes. Dentre as 14 amostras de queijo da marca C, $1(7,1 \%)$ apresentou-se contaminada com L. monocytogenes e $1(7,1 \%) \mathrm{com}$ $L$. innocua. Os queijos da marca $\mathrm{D}$ revelaram as maiores freqüências de isolamento, 14 (42,4\%), 4 (12,1\%) e 1 (3\%) amostras foram positivas para $L$. monocytogenes, $L$. innocua e $L$. grayi, respectivamente (Tabela 1).

\section{TABELA 1 - INCIDÊNCIA DE Listeria spp. EM DIFERENTES MARCAS DE QUEIJO DE COALHO INDUSTRIALIZADO E REFRIGERADO}

\begin{tabular}{|c|c|c|c|c|}
\hline \multirow{2}{*}{$\begin{array}{l}\text { Marcas de } \\
\text { queijo }\end{array}$} & \multirow{2}{*}{$\begin{array}{c}\text { Amostras } \\
\text { analisadas }\end{array}$} & \multicolumn{3}{|c|}{ Amostras positivas $^{\mathrm{a}}$ para } \\
\hline & & $\begin{array}{c}\text { L. monocytogenes } \\
\mathrm{N}^{\circ}(\%)\end{array}$ & $\begin{array}{l}\text { L. innocua } \\
\mathrm{N}^{\circ}(\%)\end{array}$ & $\begin{array}{l}\text { L. grayi } \\
\mathrm{N}^{\circ}(\%)\end{array}$ \\
\hline A & 25 & $1 \quad(4)$ & - & - \\
\hline B & 12 & - & - & - \\
\hline C & 14 & $1 \quad(7,1)$ & $1 \quad(7,1)$ & - \\
\hline D & 33 & $14 \quad(42,4)$ & $4 \quad(12,1)$ & $1 \quad$ (3) \\
\hline TOTAL & 84 & $16 \quad(19)$ & $\begin{array}{ll}5 & (5,95)\end{array}$ & $1 \quad(1,2)$ \\
\hline
\end{tabular}

a em cada amostra analisada foi encontrada uma ou mais espécies de Listeria.

Espécies de Listeria foram isoladas em amostras de queijo de coalho com valores de $\mathrm{pH}$ entre 5,66 e 6,37 (Tabela 2). Os valores mínimo e máximo de atividade de água das amostras foram 0,949 e 0,970, respectivamente. 
Apenas $L$. inoccua foi isolada na amostra de queijo com pH 5,66 $\left(\mathrm{C}_{10}\right)$, L. monocytogenes foi isolada na faixa de $\mathrm{pH}$ entre 5,76 e 6,37 . Considerando que o melhor desenvolvimento de $L$. monocytogenes é observado em pH entre seis e oito, o patógeno foi isolado em nove amostras cujas condições não são consideradas ótimas com relação a esse parâmetro (Tabela 2).

\section{TABELA 2 - ESPÉCIES DE Listeria ISOLADAS NOS RESPECTIVOS VALORES DE pH E ATIVIDADE DE ÁGUA (AW)}

\begin{tabular}{|c|c|c|c|}
\hline Amostra & $\mathrm{PH}$ & Aw & EspØcie \\
\hline$A_{8}$ & 6,37 & 0,949 & L. monocytogenes \\
\hline $\mathrm{C}_{4}$ & 6,04 & 0,970 & L. monocytogenes \\
\hline$C_{10}$ & 5,66 & 0,968 & L. innocua \\
\hline $\mathrm{D}_{1}$ & 6,10 & 0,958 & L. monocytogenes e L. innocua \\
\hline $\mathrm{D}_{2}$ & 6,16 & 0,952 & L. monocytogenes e L. Innocua \\
\hline $\mathrm{D}_{3}$ & 6,04 & 0,955 & L. monocytogenes, L. innocua e L. grayi \\
\hline $\mathrm{D}_{5}$ & 5,84 & 0,954 & L. monocytogenes \\
\hline $\mathrm{D}_{6}$ & 5,83 & 0.954 & L. monocytogenes \\
\hline $\mathrm{D}_{7}$ & 5,91 & 0,952 & L. monocytogenes \\
\hline $\mathrm{D}_{8}$ & 5,80 & 0,952 & L. monocytogenes \\
\hline $\mathrm{D}_{9}$ & 5,88 & 0,953 & L. monocytogenes \\
\hline $\mathrm{D}_{10}$ & 5,76 & 0,955 & L. monocytogenes \\
\hline$D_{11}$ & 5,83 & 0,955 & L. monocytogenes \\
\hline$D_{12}$ & 6,05 & 0,953 & L. monocytogenes \\
\hline$D_{13}$ & 6,06 & 0,954 & L. monocytogenes e L. innocua \\
\hline $\mathrm{D}_{14}$ & 5,90 & 0,952 & L. monocytogenes \\
\hline$D_{15}$ & 5,90 & 0,951 & L. monocytogenes \\
\hline
\end{tabular}

De acordo com RYSER e MARTH (1991), o pH mínimo no qual L. monocytogenes apresenta desenvolvimento é marcadamente influenciado pela temperatura e pelo tipo de ácido presente no meio. Citam que a temperatura de refrigeração confere a $L$. monocytogenes certa proteção contra o estresse causado pelo baixo $\mathrm{pH}$ em alimentos. 
Conforme PARISH e HIGGINS (1989), apud RYSER e DONNELLY (2001), L. monocytogenes sobreviveu em suco de laranja com pH 3,6 durante 21 dias e em pH 5,8 por mais de 90 dias, quando o suco foi mantido a $4^{\circ} \mathrm{C}$. Os autores observaram, ainda, redução na resistência à acidez com o aumento da temperatura de estocagem.

L. monocytogenes foi isolada em queijos de coalho com atividade de água entre 0,949 e 0,970. Segundo FRANCO e LANDGRAF (1996) já foi relatada a sobrevivência de $L$. monocytogenes a $4^{\circ} \mathrm{C}$, durante pelo menos 132 dias, em caldo tripticase de soja com atividade de água de aproximadamente 0,83 (valor extremamente baixo para a grande maioria das bactérias).

Os fatores intrínsecos e extrínsecos dos alimentos devem ser considerados no estudo do desenvolvimento de L. monocytogenes, visando avaliar seu potencial em condições adversas. SOUSA (2002) evidenciou o isolamento desse patógeno em duas amostras de queijo de coalho artesanal (armazenado em temperatura ambiente), uma com $a_{w}$ de 0,936 e pH 6,5 e outra com $\mathrm{a}_{w}$ 0,938 e pH 5,51.

A incidência de Listeria monocytogenes encontrada nesse estudo $(19 \%)$ foi considerada bastante alta à semelhança dos valores verificados por outros pesquisadores que analisaram diferentes tipos de queijo (GUERRA, McLAUCHLIN e BERNARDO, 2001; RUDOLF e SCHERER, 2000). Ocorrências em torno de $10 \%$ foram relatadas por SILVA, 1997; GUERRA e BERNARDO, 1999a e DESTRO, SERRANO e KABUKI, 1991. Incidência bem menor foi encontrada por SOUSA (2002) que detectou a presença de L. monocytogenes em apenas 2 das 70 amostras de queijo de coalho artesanal (comercializados em temperatura ambiente em Fortaleza) analisadas.

SOUSA et al. (2000) ao analisarem 30 amostras de queijo de coalho, comercializados na cidade de João Pessoa, observaram que $50 \%$ estavam contaminadas com espécies de Listeria.

L. monocytogenes foi a espécie predominante nas 17 amostras de queijo de coalho contaminadas. Resultados semelhantes foram encontrados por GUERRA e BERNARDO (1999a), que também utilizaram caldo LEB-UVM 1 na etapa de enriquecimento seletivo de queijos. Pesquisadores como SILVA (1997) e SOUSA et al. (2000) 
encontraram prevalência de L. innocua. Para SOUSA (2002), a espécie predominante em queijo de coalho artesanal (comercializado a temperatura ambiente em Fortaleza) foi $L$. welshimeri.

Todas as amostras de queijo analisadas estavam envolvidas por filme plástico aderente, possibilitando a criação de condições de microaerofilia. Segundo GUERRA e BERNARDO (1999a) tal condição, conjugada ao armazenamento prolongado em baixas temperaturas, favorece a multiplicação de Listeria nos queijos embalados e refrigerados. Além disso, GUERRA et al. (2001) ao analisarem queijos refrigerados com e sem embalagem isolaram $L$. monocytogenes apenas em queijos pré-embalados. Nessas condições, o consumidor estaria exposto à grande quantidade do patógeno, mesmo que a contagem inicial do organismo fosse baixa. Além dos aspectos quantitativos, o potencial virulento do isolado deve ser considerado (HOF e ROCOURT, 1992). RYSER e MARTH (1991) citam estudos indicando que a estocagem refrigerada pode induzir a virulência das cepas de Listeria monocytogenes.

A alta incidência de Listeria monocytogenes encontrada deve alertar as autoridades sanitárias para o perigo que representa o consumo desse tipo de queijo por parte da população suscetível. O risco de queijo de coalho veicular listeriose é realçado pelo fato de tal produto ser consumido sem receber tratamento térmico posterior. Considerando que a legislação brasileira determina que todos os queijos produzidos industrialmente devem ser preparados com leite pasteurizado (processo eficiente na destruição de Listeria), a contaminação de tais produtos ocorre em etapas pós-processamento ou resulta de subprocessamento.

De acordo com KOZAK (1986) apud FIGUEIREDO (2000), o ambiente industrial é o principal fator que contribui para a contaminação póspasteurização de laticínios e outros tipos de alimentos. FIGUEIREDO (2000) pesquisou L. monocytogenes em linha de processamento de leite pasteurizado tipo "C". Constatou por meio de tipagem molecular, pelo método RAPD, que a contaminação das amostras de leite pasteurizado ocorreu pós-pasteurização. RYSER e MARTH (1991) afirmam que, em geral, as indústrias não informam à comunidade científica sobre a contaminação de suas instalações e produtos com Listeria, receando a publicidade negativa e perdas monetárias. 
Em indústrias de carnes e laticínios já foi detectada a presença de biofilmes causados por espécies de Listeria (ROCOURT e COSSART, 1997). Estudos realizados por BLACKMAN e FRANK (1996) revelaram a habilidade de Listeria monocytogenes de acumular-se em materiais comumente encontrados na indústria de alimentos (aço, aço inoxidável, vidro e borracha). UNNERSTAD et al. (1996) afirmaram que Listeria monocytogenes pode sobreviver em indústrias de alimentos por, no mínimo, sete anos. Conforme PITT et al. (1999), os biofilmes funcionam como reserva de nutrientes e ainda protegem o microrganismo da ação de agentes sanitizantes.

Devido à natureza ubiqüitária da L. monocytogenes, eliminar completamente sua presença no ambiente industrial é praticamente impossível. Entretanto, a prevenção da contaminação dos alimentos pode ser conseguida mediante rigoroso controle da matéria-prima, processamento adequado e adoção de programa de higiene e sanificação no ambiente industrial.

3.2 DESEMPENHO DO TECRA LISTERIA VISUAL IMMUNOASSAY (LISVIA) MODIFICADO NA DETECÇÃO DE Listeria spp.

\subsubsection{Detecção de Listeria spp. por meio do TECRA LISVIA modificado}

Das 84 unidades das quatro marcas $(A, B, C$ e $D)$ de queijo de coalho analisadas com o Tecra LISVIA modificado, 1 (4\%) amostra da marca A e $8(24,2 \%)$ da marca D estavam contaminadas com Listeria spp. (9 amostras positivas). Listeria spp. não foi detectada em 12 e 14 amostras das marcas $\mathrm{B}$ e $\mathrm{C}$, respectivamente (Tabela 3 ).

As culturas positivas de nove amostras de queijo de coalho (detectadas pelo Tecra LISVIA modificado) foram confirmadas por testes culturais e bioquímicos, sendo Listeria monocytogenes a única espécie isolada.

Os resultados obtidos superam os encontrados por BORGES et al. (2000), usando Tecra LISVIA, que observaram a presença de Listeria spp. em $7 \%$ das amostras de queijo coalho procedentes do Ceará e do Rio Grande do Norte. 


\section{TABELA 3 - DETECÇÃO DE Listeria spp. EM AMOSTRAS DE QUEIJO DE COALHO UTILIZANDO O TECRA LISVIA MODIFICADO}

\begin{tabular}{cccc}
\hline $\begin{array}{c}\text { Marcas de } \\
\text { queijo }\end{array}$ & $\begin{array}{c}\mathrm{N}^{\circ} \text { de Amostras } \\
\text { analisadas }\end{array}$ & $\begin{array}{c}\text { Listeria spp. } \\
\mathrm{N}^{\circ}\end{array}$ & $\begin{array}{c}(\%) \\
\text { A }\end{array}$ \\
$\mathrm{B}$ & 25 & 1 & $(4)$ \\
$\mathrm{C}$ & 12 & - \\
$\mathrm{D}$ & 14 & & - \\
\hline TOTAL & 33 & 8 & $(24,2)$ \\
\hline
\end{tabular}

*substituição do caldo LEB por LEB-UVM 1

A presença do gênero Listeria em determinado produto indica processamento inadequado e risco de contaminação com Listeria monocytogenes, face às idênticas condições de crescimento de espécies patogênicas e não-patogênicas. Tal fato ressalta a importância de avaliar a ocorrência do gênero Listeria em alimentos (RUDOLF e SCHERER, 2000).

\subsubsection{Presença de Listeria spp. não-detectada pelo TECRA LISVIA modificado}

Obteve-se resultado negativo para 75 amostras de queijo de coalho na leitura visual do Tecra LISVIA modificado. Após plaqueamento, isolamento de colônias suspeitas e caracterização bioquímica foram encontradas duas amostras positivas para Listeria no queijo da marca C e 6 da marca $D$ (Tabela 4). Listeria monocytogenes foi isolada em 6 amostras da marca D e 1 da marca C.

Inúmeras pesquisas têm revelado alto índice de correlação entre o teste rápido e os métodos culturais (ATRACHE et al., 1989; NOAH et al., 1991), quando diferentes porções do alimento são analisadas simultaneamente pelos dois métodos. Nesse estudo, a mesma porção do alimento previamente enriquecida foi avaliada, simultaneamente, pelo teste rápido e pelo método tradicional de cultivo. 
O caldo LEB-UVM 1, embora recomendado para detecção de Listeria spp. em carnes, tem proporcionado bons resultados em leites e queijos (RYSER e MARTH, 1991; GUERRA e BERNARDO, 1999a; WARBURTON et al.,1992 FIGUEREDO, 2000). Além disso, o método do Health Protection Branch do Canadá (FARBER et al, 1994), aplicável para todas as categorias de alimentos, utiliza o caldo LEB-UVM 1 como meio de enriquecimento primário. Outro aspecto importante é que a própria estocagem refrigerada do produto logo após a produção apresenta caráter seletivo para espécies de Listeria. ROSENOW e MARTH (1987) apud RYSER e MARTH (1991), ao estudarem o crescimento de Listeria em laticínios, observaram que a população de L. monocytogenes atingiu contagens de $10^{7}$ a $10^{9} \mathrm{UFC} / \mathrm{mL}$, após incubação dos produtos a $4^{\circ} \mathrm{C}$ por 30 a 45 dias ou a $8^{\circ} \mathrm{C}$ por $11-14$ dias. Deve-se ressaltar que o prazo de validade dos queijos analisados variava de 45 a 60 dias em temperaturas de refrigeração.

\section{TABELA 4 - ISOLAMENTO DE Listeria spp. E L. Monocytogenes EM AMOSTRAS NEGATIVAS NO TECRA LISVIA MODIFICADO}

\begin{tabular}{|c|c|c|}
\hline $\begin{array}{c}\text { Marcas de } \\
\text { queijo }\end{array}$ & $\begin{array}{c}\mathrm{N}^{\circ} \text { de Amostras } \\
\text { analisadas }\end{array}$ & $\begin{array}{c}\text { Listeria spp. } \\
\mathrm{N}^{\circ}(\%)\end{array}$ \\
\hline A & 24 & - \\
\hline B & 12 & - \\
\hline C & 14 & $2(14,3)$ \\
\hline D & 25 & $6 \quad(24,2)$ \\
\hline TOTAL & 75 & $8 \quad(10,7)$ \\
\hline
\end{tabular}

É improvável que o procedimento de enriquecimento (adotado nesse estudo) não tenha permitido que a população de Listeria tenha atingido os níveis detectáveis pelo ensaio de ELISA, que segundo BEUMER e BRINKMAN (1989) apud NOAH et al. (1991) para alguns alimentos deve ser de $10^{6}$ células por $\mathrm{mL}$. O teste rápido utilizado nesse estudo não foi capaz de detectar a presença de Listeria em 8 das 17 amostras contaminadas com a bactéria, provavelmente devido a falha técnica do ensaio, uma vez que um único kit foi utilizado para 96 amostras. 
A habilidade do teste de ELISA em detectar Listeria spp. depende do tipo de alimento analisado. NORRUNG et al. (1991) observaram que esse teste mostrou-se limitado na detecção de Listeria spp. em alimentos crus com baixo níveis de Listeria e elevada microbiota competidora. Todas as amostras de queijo analisadas neste estudo apresentavam o selo de inspeção federal (S.I.F.) ou estadual (S.I.E.), constando no rótulo que foram produzidas com leite pasteurizado. Contudo, vários pesquisadores (MENDES et al., 1999; BORGES et al., 2000; LEITE JUNIOR et al., 2000) têm comprovado elevada contaminação microbiana neste tipo de queijo.

\section{CONCLUSÃO}

Constatou-se elevada contaminação de queijo de coalho industrializado, comercializado sob refrigeração com L. monocytogenes.

A maioria das cepas de L.monocytogenes foi isolada em amostras de queijo com pH e $a_{w}$ abaixo das condições ótimas de seu desenvolvimento.

O TECRA Listeria Visual Immunoassay modificado não detectou a presença de Listeria spp. em 7 (46,2\%) das amostras contaminadas.

\section{Abstract}

\section{INCIDENCE OF Listeria monocytogenes IN INDUSTRIALLY MANUFACTURED REFRIGERATED "COALHO" CHEESE}

Incidence of Listeria monocytogenes in "coalho" cheese industrially manufactured and commercialized in refrigerated temperature in the city of Fortaleza, Ceará (Brazil) was evaluated. Water activity and $\mathrm{pH}$ conditions in the contaminated samples with Listeria species were also evaluated. Samples (84) of industrialized "coalho" cheese of different brands were analyzed using the modified TECRA Listeria Visual Immunoassay (LISVIA). From 84 samples, 16 (19\%) were contaminated with Listeria monocytogenes, 5 (5.9\%) with Listeria innocua and 1 (1.2\%) with Listeria grayi. Listeria monocytogenes was isolated in the $\mathrm{pH}$ range of 5.75 to 6.37 and in water activity between 0.949 e 0.970 . The TECRA LISVIA detected the presence of Listeria spp. in $9(10.7 \%)$ samples. All positives samples in the rapid test were confirmed by cultural and biochemical tests and in all samples the presence of Listeria monocytogenes was detected. The negative samples plating in the visual reading of the rapid test allowed the isolation of Listeria spp. in 8 samples and in 7 the presence of Listeria monocytogenes was detected.

KEY-WORDS: TECRA LISVIA; Listeria Monocytogenes; COALHO CHEESE. 


\section{REFERÊNCIAS}

1 AOAC. Association of Official Analytical Chemistry. Official methods of AOAC international. $12^{\text {th }}$ ed. Washington, 1992.

2 AQUALAB. Analisador de atividade de água para avaliar biodegradação (alimentos e fármacos): Modelo CX-2. Decagon Devices, Inc. 950 NE Nelson Court Pullman, WA 99163 USA, 2001.

3 AQUINO, F. T. M. Produção de queijo de coalho no Estado da Paraíba: acompanhamento das características físico-químicas do processamento. 1983. 74 p. Dissertação (Mestrado) em Ciência e Tecnologia de Alimentos - Centro de Tecnologia, Universidade Federal da Paraíba, João Pessoa.

4 ATRACHE, V. et al. A rapid enzyme immunoassay for detection of Listeria in food and environmental samples. In: SEMINARY ON MODERN MICROBIOLOGICAL METHODS FOR DAIRY PRODUCTS, 1989, Santander, Spain. Proceedings... Brussels, Belgium: International Dairy Federation, 1989. p. 346-348 (International Dairy Federation Special Issue, n.8901).

5 BANNISTER, B. A. Listeria monocytogenes meningitis associated with eating soft cheese. Journal of Infection, v.15, p. 165-168, 1987.

6 BECKERS, H. J., SOENTORO, P. S. S.; DELFGOU-VAN ASH, E. H. H. The occurrence of Listeria monocytogenes in soft cheese and raw milk and its resistence to heat. International Journal of Food Microbiology, v. 4, p. 249- 256, 1987.

7 BENEVIDES, S. D. Comportamento do queijo de coalho produzido com leite cru e pasteurizado, maturado a temperatura ambiente e sob refrigeração. Fortaleza, 1999. 132 p. Dissertação (Mestrado) em Tecnologia de Alimentos - Departamento de Tecnologia de Alimentos, Universidade Federal do Ceará .

8 BLACKMAN, I. C.; FRANK, J. F. Growth of Listeria monocytogenes as a biofilm on various food-processing surfaces. Journal of Food Protection, v. 59, n. 8, p. 827- 831, 1996.

9 BORGES, M. F. et al. Pathogenic and indicator microrganisms isolated from "coalho" cheese produced in Ceará and Rio Grande do Norte, states of Brasil. In: CONGRESSO LATINOAMERICANO DE MICROBIOLOGIA DE ALIMENTOS, 6. 2000, Buenos Aires. Anais... Buenos Aires: LAS-ICMSF, 2000. p. 116.

10 DALTON, C. B. et al. An outbreak of gastroenteritis and fever due to Listeria monocytogenes in milk. New England Journal of Medicine, v. 336, n. 2, p. 100-105, 1997.

11 DESTRO, M. T.; SERRANO, A. M.; KABUKI, D. Y. Isolation of Listeria species from some Brazilian meat and dairy products. Food Control, v. 2, p. 110-112, 1991. 
12 DILLON, R. M.; PATEL, T. R. Listeria in seafoods: a review. Journal of Food Protection, v. 55, p. 1009-1015, 1992.

13 FARBER, J. M.; PETERKIN, P. I. Listeria monocytogenes, a food-borne pathogen. Microbial Reviews, v.55, n.3, p. 476-511, 1991.

14 FARBER, J. M.; WARBURTON, D. W.; BABIUK, T. Isolation of Listeria monocytogenes from all food and environmental samples. Otawa: Health Protection Branch, Government of Canadá, 1994. 16 p. (MFHPB-30).

15 FEITOSA, T. Estudos tecnológicos, físico-químicos, microbiológicos e sensoriais do queijo de coalho do estado do Ceará. 1984. 96 p. Dissertação (Mestrado em Tecnologia de Alimentos), Departamento de Tecnologia de Alimentos, Universidade Federal do Ceará, Fortaleza.

16 FIGUEIREDO, E. A. T. Ocorrência do gênero Listeria e avaliação da diversidade genética de Listeria monocytogenes através de Random Amplified Polymorphic DNA (RAPD) e sua distribuição em linha de processamento de leite pasteurizado tipo "C". São Paulo, 2000. 100 p. Tese (Doutorado em Microbiologia) - Instituto de Ciências Biomédicas, Universidade de São Paulo.

17 FLORENTINO, E. R.; MARTINS, R. S. Características microbiológicas do queijo de coalho produzido no estado da Paraíba. Higiene Alimentar, v.13, n. 59, p.43-48, 1999.

18 FRANCO, B. D. G. H.; LANDGRAF, H. Microbiologia dos alimentos. São Paulo: Atheneu, 1996. $182 \mathrm{p}$.

19 GUERRA, M. M.; BERNARDO, F. M. A. Ocorrência natural de Listeria spp. em queijos Alentejanos. Revista Portuguesa de Ciências Veterinária, v.94, n.530, p. 63-74, 1999a.

20 GUERRA, M. M.; BERNARDO, F. M. A. Relevância sanitária de Listeria monocytogenes nos produtos lácticos. Revista Portuguesa de Ciências Veterinária, V. 94 , n. 530, p. 142-148, 1999b.

21 GUERRA, M. M., McLAUCHLIN, F. A.; BERNARDO, F. M. A. Listeria in ready-toeat and unprocessed foods produced in Portugal. Food Microbiology, v. 18, p. 423-429, 2001.

22 HOF, H.; ROCOURT, J. Is any strain of Listeria monocytogenes detected in food a health risk? International Journal of Food Microbiology, v.16, p.173182, 1992.

23 JOHNSON, J. L.; DOYLE, M. P.; CASSENS, R. G. Listeria monocytogenes and other Listeria spp. in meat and meat products: a review. Journal of Food Protection, v.53, p. 81-9, 1990.

24 LEITE JÚNIOR, A. F. S. et al. Qualidade microbiológica do queijo de coalho comercializado à temperatura ambiente ou sob refrigeração, em Campina 
Grande-PB. Higiene Alimentar, v. 14, n.73, p. 53-59. 2000.

25 MENDES, E. S. et al.. Staphylococcus aureus, Salmonella sp. e coliformes em queijos de "coalho" comercializados em Recife. Higiene Alimentar, v. 13, n.66/67, p. 122-126, 1999.

26 NASSU, R. T. et al. Diagnóstico das condições de processamento e qualidade microbiológica de produtos regionais derivados do leite produzido no estado do Rio Grande do Norte. In: CONGRESSO NACIONAL DE LATICÍNIOS, 17., 2000, Juiz de Fora. Anais... Juiz de Fora: Dairy Journal, 2000. p. 121-126

27 NOAH, C. W.; RAMOS, N. C.; GIPSON, M. V. Efficiency of two commercial ELISA kits compared with the BAM cultural method for detecting Listeria in naturally contaminated foods. J. Assoc. Off. Anal. Chem., v.74, n.5, p. 819-821, 1991.

28 NORRUNG, B. et al. Evaluation of an ELISA test for detection of Listeria spp. Journal of Food Protection, v.54, n. 10, p. 752-755. 1991.

29 PEREIRA, M. L.; ROCOURT, J. Listeria monocytogenes: uma revisão sobre aspectos taxonômicos, importância médica e em alimentos. Higiene Alimentar, v. 7, n.26, p. 7-13, 1993.

30 PITT, W. M.; HARDEN, T. J.; HULL, R. R. Listeria monocytogenes in milk and dairy products. The Australian Journal of Dairy Tecnology, v. 54, p. 49-65, Apr., 1999.

31 RIEDO, F. X. et al. A pont-source foodborne listeriosis outbreak: documented incubation period and possible mild illness. Journal Infection Disease, $v$. 170, p. 693-696, 1994.

32 ROCOURT, J.; COSSART, P. Listeria monocytogenes. In: DOYLE, M. P.; BEUCHAT, L. R.; MONTEVILLE, T. J. Food microbiology: fundamentals and frontiers. Washington: ASM, 1997. p.337-352.

33 BEUCHAT, L. R.; MONTEVILLE, T. J. Food microbiology: fundamentals and frontiers. Washington: ASM, 1997. p.337-352.

34 RUDOLF, M.; SHERER, S. High incidence of Listeria monocytogenes in European red smear cheese. International Journal of Food Microbiology, v. 63, n. 2001, p. 91-98, 2000.

35 RYSER, E. T.; DONNELY, C. W. Listeria. In: DOWNES, F. P.; ITO, K. Compendium of methods for the microbiological examination of foods. $4^{\text {th }}$ ed. Washington: American Public Health Association, 2001. p.343-356. Chapter 36.

36 RYSER, E. T.; MARTH, E. H. Listeria, listeriosis and food safety. New York: Marcel Dekker, 1991. 631 p.

37 RYSER, E. T.; MARTH, E. H. Behavior of Listeria monocytogenes during the 
manufacture and ripening of Cheddar Cheese. Journal of Food Protection, v. 50 , p. $7-13,1987$ a.

38 RYSER, E. T.; MARTH, E. H. Fate of Listeria monocytogenes during the manufacture and ripening of camembert cheese. Journal of Food Protection, v. 50 , n. 5 , p. $372-378,1987$ b.

39 RYSER, E. T., MARTH, E. H.; DOYLE, M. P. Survival of Listeria monocytogenes during the manufacture and storage of cottage cheese. Journal of Food Protection, v. 48, n.9, p. 746-750, 1985.

40 SANTOS, F. A.; NOGUEIRA, N. A. P.; CUNHA, G. M. A. Aspectos microbiológicos do queijo tipo "coalho" comercializado em Fortaleza - Ceará. Boletim do CEPPA, v.13, n.1, p.31-36, jan. /jun. 1995.

41 SEBRAE. Projeto melhoria da qualidade do queijo de coalho produzido no Ceará. Fortaleza, 1998.

42 SILVA, M. C. D. Ocorrência e avaliação de métodos para detecção e caracterização de Listeria isoladas de queijo. Rio de Janeiro, 1997. 117 p. Tese (Doutorado em Microbiologia), Instituto de Microbiologia, Universidade Federal do Rio de Janeiro.

43 SOUSA, R. S. Incidência de Listeria monocytogenes em queijo tipo coalho artesanal comercializado a temperatura ambiente em Fortaleza. Fortaleza, 2002. 90 p. Dissertação (Mestrado em Tecnologia de Alimentos), Departamento de Tecnologia de Alimentos, Universidade Federal do Ceará.

44 SOUSA, S.; LIMA, A. W. O.; SOUSA, C. P. Isolamento de espécies de Listeria em queijo de massa crua tipo coalho comercializada na cidade de João Pessoa -PB. In:CONGRESSO BRASILEIRO DE CIÊNCIA E TECNOLOGIA DE ALIMENTOS, 17., 2000, Fortaleza. Anais... Fortaleza: CBCTA, 2000. p. 4.145.

45 TECRA LISTERIA VISUAL IMMUNOASSAY. Manual do fabricante. São Paulo: Madasa do Brasil, 2000. 13 p.

46 UNNERSTAD, $\mathrm{H}$. et al. Prolonged contamination of a dairy with Listeria monocytogenes. Milk Dairy J., v. 50, p.493-499, 1996.

47 WARBURTON, D. W. et al. Comparison of methods for optimum detection of stressed and low levels of Listeria monocytogenes. Food Microbiology, v. 9, p. 127-145, 1992.

48 WHER, H. M. Listeria monocytogenes: a current dilemma. J. Assoc. Off. Anal. Chem. v. 70, p. 769-772, 1987. 